\title{
The Research of Memory Model Based on Granular Computing
}

\author{
$\mathrm{Xu}$ Wenchao \\ Academy of Armored Forces Engineering \\ AAFE \\ Beijing, China \\ moonhills@126.com, \\ Wang Enjuan \\ Vocational Education Center of Shandong Yishui \\ VECSY \\ Shandong, China \\ Wang-enjuan@163.com
}

\author{
Xue Qing \\ Academy of Armored Forces Engineering \\ AAFE \\ Beijing, China \\ xue_qing@yeah.net \\ Zheng Changwei \\ Academy of Armored Forces Engineering \\ AAFE \\ Beijing, China \\ Zhw_byh@163.com
}

\begin{abstract}
To simulate human memory, this paper introduces granular computing into modeling of memory, establishes a three order system, divides the memory model into instantaneous memory module, working memory module and the long-term memory module, filters out the memory information, granular coding the information that be noted, analysis of the characteristics of long-term memory information, proposes the long-term memory information retrieval method, researches the forgetting.
\end{abstract}

Keywords-Memory model; Granular coding; Clues; Forgetting

\section{INTRODUCTION}

Memory is the psychological process of accumulation and preservation in mind individual experience [1]. The unit of processing memory information is chunk, the chunk combine levels of code into a single high-level code. The granule in granular computing is some of the individual (elements, points, etc.) formed blocks through the indistinguishability relationship, similarity relationship, proximity or functionality relationships [2]. Granular computing has structural features in problem solving and information processing, which similarly of the encoding process of memory. The paper proposes the memory model based on granular computing, introduces the ideas and methods of granular computing into the construction of memory models.

\section{THE THIRD-ORDER SYSTEM OF MEMORY MODEL}

Based on storage capacity and storage time, this article will be divided into three modules perception memory model: the instantaneous memory modules, working memory modules and the long-term memory module. Each module is divided into storage unit and execution unit.

The Instantaneous Memory Module

Instantaneous memory is the beginning of the memory, it is the feeling of a more primitive form, is the registration of memory model further processing the outside information. Instantaneous memory encoding depends on the physical characteristics, it has a distinct image. When the environment effect disappeared, instantaneous memory module offers the possibility for further processing the information. Instantaneous memory with large storage capacity, but most of the information disappearing because of not processed, only the ones noticed by individuals are further processed and access to working memory. Instantaneous memory preserved a short time.

The Working Memory Module

Working memory module is the intermediate links of the instantaneous memory and long-term memory transition. The greatest feature of working memory is the limited capacity, its storage capacity is $7 \pm 2$ [3], whereby the paper set the working memory module storage capacity 7 , the unit is chunk. Implementation unit of working memory module can get instantaneous memory and long-term memory information, and complete the coding.

the Long-term Memory Module

Long-term memory module stores all the knowledge and experience, afford all knowledge for the necessary activities. Long-term memory module information is stored for a long time, capacity is not restricted, most of the content comes from the working memory process[1].

\section{THE INFORMATION STREAM OF MEMORY MODEL}

The information stream of memory model shown as Figure 1:

The information stream of memory model is divided into three processes as shown:

(1) Instantaneous memory turns into working memory

The information stream of instantaneous memory is huge, plenty of outside information come into instantaneous memory module, part of them to be noticed and comes into working memory, a little directly comes into long-term memory, most of them disappears.

(2) Working memory turns into long-term memory

There are two conditions that working memory turns into long-term memory: repetition and reasonable code.

(3) Working memory module gets information from the long-term memory

Working memory needs to get long-term memory knowledge in the encoding process. 


\section{THE REALIZATION OF MEMORY MODEL BASED ON GRANULAR COMPUTING}

\section{A. instantaneous memory storage and operation}

The instantaneous memory module numbers the coming information and adds store timer, the time is set $2 \mathrm{~s}$. The instantaneous memory module deletes the information that the timer comes to the default time.

The main function of the instantaneous memory module is classification of information,[4] for one hand it selects the information could come into working memory, for the other, it determines the order. The paper define these could directly come into working memory as the first class information, and the rest as second class information.

The principle of the first class information is singularity and intensity of the information.[5] Singularity of the information is unusual characteristics. For example, the first time one saw a panda, it is singularity. When the intensity of information beyond acceptance, such as sound of explosions. The information also comes into working memory directly.

Instantaneous memory module grants the second class information priority, high-priority information comes into working memory priority. Priority rules are as follows:

(1) Relate to the current activities

(2) Relate to professional or interest

(3) Familiar to the individual

The existence of three rules is equivalent, the priority plus 1 when comfort one rule, high priority first enter the working memory module, when the priority is equal, earlier the information comes into the instantaneous memory earlier it comes into working memory.

The instantaneous memory module informs the queue of formation of memory information as figure 2 . In figure 2 , the $G_{I D}$ show the priority $\mathrm{j}$ and ID.

\section{B. Granular Encoding of Memory Information}

Working memory module encodes the information based on granular computing, calculates this information into the memory information granule. Some granule will come into long-term memory module as memory information. The paper describes memory information granule with a triple group $\mathrm{G}=(\mathrm{IG}, \mathrm{EG},(\mathrm{SFG}, \mathrm{NFG}))$, in which, IG as the content of memory information, EG as the extension of memory information, SFG as the special transfer function between the content and the extension, NFG as general transfer function

Memory information granule's structure reflects its three basic attributes, as follow:

Show the internal attributes of the interaction between elements of memory information granule.

Show the external attributes of interactions between the memory information granules

Reveal the granule environment context properties of memory information granules.

The content IG of memory information granule $G$ is a set that includes the properties of the granule called property element (AE) and content granule called granule elements (GE). IG can be formalized as follows:

$$
I G=\left\{A E_{1}, A E_{2}, A E_{3}, \cdots, G E_{1}, G E_{2}, G E_{3}, \cdots\right\}
$$

Extension EG is associative list of memory information granule $\mathrm{G}$, which stores the granule-related indexes and related degrees. EG can be formalized as follows

$$
E G=\left\{\left(G_{i}, u_{i}\right),\left(G_{j}, u_{j}\right),\left(G_{k}, u_{k}\right), \cdots\right\}
$$

SFG denotes $(\mathrm{EG} \rightarrow \mathrm{IG})$, NFG denotes (IG $\rightarrow \mathrm{EG})$, both include a set of rules.

Reasonable encoding of information is a prerequisite, but not sufficient condition come into long-term memory. Chaos information may be come into the working memory module for some reason, but it can not be encoded and can not enter the long-term memory.

The condition that granule enter into long-term memory is repeated. Chris and Watkins' study shows that only the mechanical repetition cannot enhance memory, fine repeat is an important condition for working memory's storage. [1] Therefore, the paper agrees that only the granule establishes reliable extension and repeated can enter into the long-term memory.

Not only the capacity is limited of working memory, but also the duration of storage.[6] If one granule cannot enter into long-term memory, the working memory module will remove it.

\section{The long-term memory information}

Memory is a kind of mental activity, the difference between memory and storage is memory information is dynamic, and will occur forgetting.

1) The amalgamation of properties of long-term memory granule

Long-term memory information is interrelated and mutually reinforced. Long-term memory module can link two pieces of information to form a combined granule. As shown in figure 3 , the property A4 of granule A supplement property $\mathrm{B}$ lof granule $\mathrm{B}$, long-term memory module can merge the two properties to the $\mathrm{B}$

2) access to information of long-term memory granules

The key to access to information is clues,[7] clues may be a property of a certain piece of information, may also be a time series, or even a vague description. People often have this experience, one can finally in retrospect through constant reminder, where the prompt is clue.

when the clue $\mathrm{C}$ is given, access granules from longterm memory information is as follows:

$$
A=\left\{G_{i} \mid G_{i} \supset C\right\}
$$

\section{3) Forgetting}

The contents of memory can not maintain or have difficulty of extraction is forgetting [1]. Forgetting memory affected by the character of the information, encoding level and other factors. This paper agrees that the information in long-term memory is not lost, the reason of forgetting is the 
link between memory granule and clues weakened or disappeared.[8] Therefore, the forgetting information can be remembered by continually prompted with other clues. Prompt can reestablish links between granule and clues.

\section{FORESEE}

The paper do research to the three orders system of memory, introduce the granular computing into the foundation of memory model, analysis the encoding and storage of memory information. It is important that establish a reality memory model to virtual man research, more work need be done about realizing the memory model based on granular computing.

\section{REFERENCES}

[1] Meng Zhaolan. General Psychology [M]. Beijing: Beijing University Press, 1994
[2] Zadeh LA, Fuzzy Logic = Computing with Words, IEEE Trans. On Fuzzy Systems, Vol.4, 1996, pp.103-111.

[3] Miller, The Magical Number $7 \pm 2$ : our information processing capacity constraints, 1956

[4] I.Kanter,H.Sompolinsky. Associative recall of memory without errors. Phys. Rev. A, 1987, 35:380-392.

[5] Quillian M R. Semantic memory [C]. In: Minsky M eds.Semantic Information Processing. Cambridge: MITPress.1968.

[6] Schank R.Dynamic Memory: ATheory ofReminding andLearning in Computers andPeoples[M]. Cambridge University Press, 1982.

[7] Rosenfield I.The Invention ofMemory: ANewTheory ofthe Brain[M]. NewYork:Basic Books Inc, 1988.

[8] Huang Y M,Chen J N,Kuo Y H,et al.An intelligent humanexpert forum system based on fuzzy information retrieval technique[J].Expert Systems with Applications,2008,34(1):446 458.

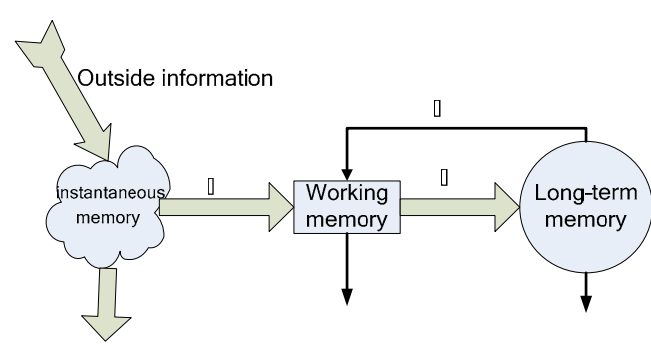

Figure1 information stream of memory model

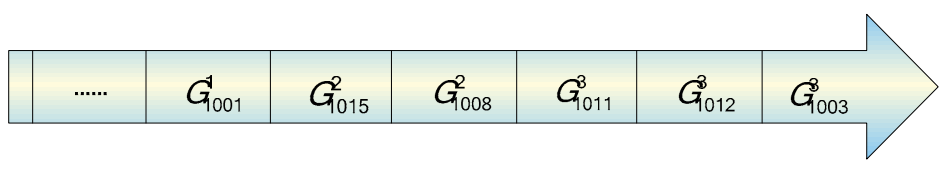

Figure 2 information queue

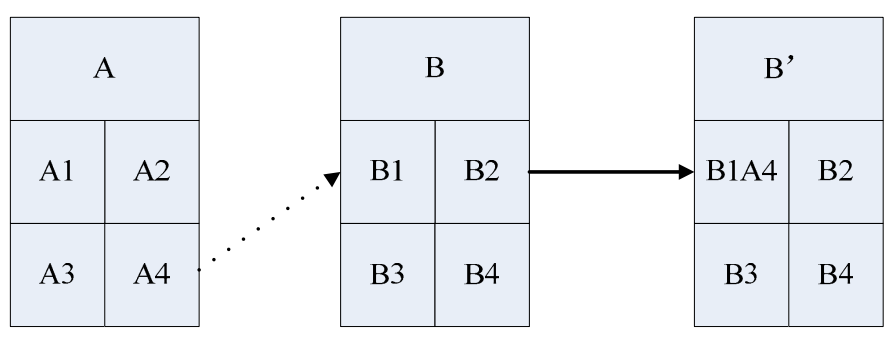

Figure 3 The amalgamation of properties of long-term memory granule 